\begin{tabular}{|c|c|}
\hline $\begin{array}{c}\text { European Association for the } \\
\text { Development of Renewable Energies, Environment } \\
\text { and Power Quality (EA4EPQ) }\end{array}$ & $\begin{array}{c}\text { International Conference on Renewable Energies and Power Quality } \\
\text { (ICREPQ'12) }\end{array}$ \\
Santiago de Compostela (Spain), 28th to 30th March, 2012
\end{tabular}

\title{
A Parallel Approach for Real-Time Power Flow in Distribution Networks
}

\author{
Rafael G. Milbradt”, Luciane N. Canha ${ }^{\#}$, Paulo R. Pereira ${ }^{*}$, Alzenira R. Abaide ${ }^{\#}$, Lukas Neusser", \\ Sandro R. Schmaedecke* and Lidia M. Garcia

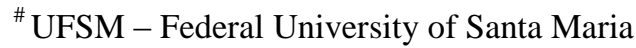 \\ Santa Maria, RS, Brazil \\ * CEEE-D - State Company of Electric Power Distribution of Rio Grande do Sul \\ Porto Alegre, RS, Brazil \\ E-mails: rmilbradt@gmail.com, Incanha@ct.ufsm.br, paulo.ricardo@cee.com.br, alzenira@ct.ufsm.br,
lukasneusser@ @otmail.com, sandroms@ceee.com.br, lidiamdg@gmail.com
}

\begin{abstract}
The new reality of smart distribution systems with use of generation sources of small and medium sizes brings new challenges for the operation of these systems. The complexity and the large number of nodes requires use of methods which can reduce the processing time of algorithms such as power flow, allowing its use in real time. This paper presents a known methodology for calculating the power flow in three phases using backward/forward sweep method, and also considering others network elements such as voltage regulators, shunt capacitors and sources of dispersed generation PV and PQ. After that, new elements are introduced that allow the parallelization of this algorithm and an adequate distribution of work between the available processors. The algorithm was implemented using a multi-tiered architecture which allows its use even in environments with low processing power, such as smartphones and tablets, and the processing times were measured in many network configurations and compared with the same algorithm in the serial version.
\end{abstract}

\section{Key words}

Distribution Power Flow, Real-time Power Flow, Distribution Automation, Dispersed Generation, Parallel Algorithm.

\section{Introduction}

The evolution of electric power systems and the requirements of the society for it, are bringing these new concepts for distribution systems such as smart metering, dispersed generation, demand-side management, microgeneration, electric vehicles, among others. In contrast, the operation of a distribution system in this way will become extremely complex, making it necessary to analyze the network more efficient, comprehensive and in real-time. It must be considered that the requirements for power quality will increase, making necessary some immediate interventions in the system to avoid the loss of the quality indicators.
The complexity of a distribution network and its large number of nodes, especially the need to consider the adjacent networks to a feeder makes the processing load for the analysis algorithms potentially large. With the introduction of remote measurement in primary distribution networks there is the possibility of real-time analysis, but the algorithms need to be optimized to a fast execution, which can be called "real time".

The traditional methods for calculating the power flow, that are widely used in transmission systems, as NewtonRaphson [1] and Fast Decoupled [2], typically do not have a good performance when applied to distribution systems. Several peculiarities of these networks such as the low ratio $\mathrm{R} / \mathrm{X}$, the unbalanced loads between phases, the presence of dispersed generation and the large number of nodes cause convergence problems to these algorithms.

According to [3] the method of backward/forward sweep is most suitable for purely radial systems, or even with a few loops, and has good reliability and fast convergence. About this same algorithm there are some variants as the method of sum of the currents [4] and the method of sum of powers [5].

Another know variant is in the calculation of the flow separated by phase [4] [5], rather than the single-phase also widely used in transmission systems. This approach is interesting because the unbalanced loads between phases are quite common in distribution networks because of the extensive presence of two-phase and single phase loads. On the other hand, the electrical utilities try to minimize the effects of these loads, distributing them symmetrically between the phases. However this symmetry is difficult to obtain considering also differences in the behavior of the loads.

This research is part of a project with CEEE-D electric utility, presenting a known methodology for the analysis of power flow in multi-phase primary and radial 
distribution networks, based in the method of backward/forward sweep (sum of the currents). In combination, are also being proposed changes to the basic algorithm for parallel processing, allowing a gain in processing time proportional to the number of available processors. The implementation of these algorithms was done in a multi-tiered architecture allowing access even in devices with low processing power and processing times were measured in various network configurations and related to the times of the basic serial algorithm.

\section{Multiphase Power Flow}

\subsection{Basic Algorithm}

For the implementation of the power flow, was chosen the backward/forward sweep algorithm with sum of the currents, adapted for the calculation in three-phase primary distribution networks. The loads represented by the distribution transformers were modeled as constant power loads (PQ). The network is computationally represented by a hierarchical data structure, where each node keeps a reference to the line section downstream and upstream, each line section also holds references to the nodes at start and end. This approach allows a better structuring of algorithms for navigation through the use of recursion. The access speed is similar to that obtained with the use of matrix/arrays and compared to these, dispensing an initial process of feeder levelization.

The algorithm is initialized with the values of nominal voltage magnitudes and angles for all of nodes and branches. Thereafter, begin $\mathrm{k}$ successive iterations of the following steps:

\section{1) Calculation of currents in the $P Q$ buses:}

All PQ buses of the network are covered, calculating the currents taking into account the bus voltage (1 p.u. in the first iteration) and the admittance matrix of the shunt elements. The admittances of the shunt elements are symmetrical, as well as the mutual admittances in most distribution networks are negligible.

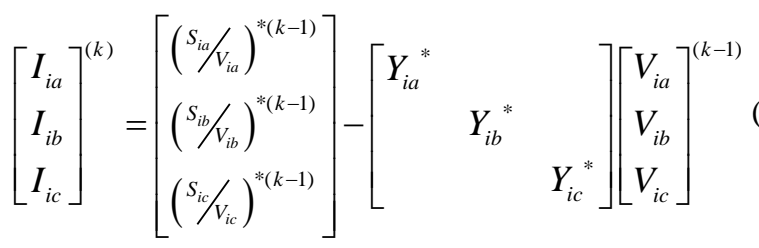

Where:

$I_{i a}, I_{i b}, I_{i c}$ are the current injections at node $i$ in the respective phases.

$S_{i a}, S_{i b}, S_{i c}$ are known power injections at node $i$.

$V_{i a}, V_{i b}, V_{i c}$ are voltages at node $i$.

$Y_{i a}, Y_{i b}, Y_{i c}$ are admittances of the shunt elements at

node $i$.

\section{2) Backward Sweep:}

Based on Kirchhoff's Law, this step consists to go through all nodes from the loads toward the source by adding the currents.

$$
\left[\begin{array}{c}
J_{l a} \\
J_{l b} \\
J_{l c}
\end{array}\right]^{(k)}=-\left[\begin{array}{c}
I_{j a} \\
I_{j b} \\
I_{c}
\end{array}\right]^{(k)}+\sum_{m \in M}\left[\begin{array}{l}
J_{m a} \\
J_{m b} \\
J_{m c}
\end{array}\right]^{(k)}
$$

Where:

$J_{l a}, J_{l b}, J_{l c}$ are the per phase currents at branch $l$. $M$ is the set of branches downstream of $l$.

\section{3) Forward Sweep:}

It consists in the calculation of the voltage magnitude and angle, for each node in the forward direction starting from the substation. The voltage values will be used to calculate the new currents in Step 1, in the next iteration of this algorithm.

$$
\left[\begin{array}{c}
V_{j a} \\
V_{j b} \\
V_{j c}
\end{array}\right]^{(k)}=\left[\begin{array}{c}
V_{i a} \\
V_{i b} \\
V_{i c}
\end{array}\right]^{(k)}-\left[\begin{array}{lll}
z_{a a} & z_{a b} & z_{a c} \\
z_{b a} & z_{b b} & z_{b c} \\
z_{c a} & z_{c b} & z_{c c}
\end{array}\right]\left[\begin{array}{c}
J_{l a} \\
J_{l b} \\
J_{l c}
\end{array}\right]^{(k)}
$$

\section{4) Convergence Checking}

In this step is made the convergence test with the results, which is based in the comparison of voltage value at each phase for each node. If the difference in any of these voltage values between the actual iteration and the previous iteration is less than the criterion to stop, then the power flow has reached the convergence.

$$
\left[\begin{array}{c}
\Delta V_{i a} \\
\Delta V_{i b} \\
\Delta V_{i c}
\end{array}\right]^{(k)}=\left[\begin{array}{c}
V_{i a} \\
V_{i b} \\
V_{i c}
\end{array}\right]^{(k)}-\left[\begin{array}{c}
V_{i a} \\
V_{i b} \\
V_{i c}
\end{array}\right]^{(k-1)}
$$

\subsection{Dispersed Generation}

The modeling of this equipment in a power flow algorithm is a required task. Especially the sources of distributed generation in recent years have grown in number considerably. However, this growth has become a challenge for the operation of the distribution system and the proper modeling and analysis of the network through the power flow in real time can be an important ally.

The sources of dispersed generation can be modeled in the following ways:

1) PQ Buses: buses where the active and reactive power are constants;

2) PV Buses: buses where the active power and voltage are constants. 
PQ buses are easy to implement in the Power Flow and requires no further treatment. They are inserted in steps of calculation as power injections, with the opposite sign of loads.

The PV buses have constant values of voltage and real power, since the reactive power can vary between a minimum and maximum. It is necessary a new step in every iteration of the algorithm, this is made by calculating the required reactive power injection in the network so the voltage at that node stay equal to the voltage set to this PV bus [6][7]. As the voltage at node changes with each sweep of the algorithm, the injection of reactive power will also changes, therefore this criterion also becomes part of the step where the convergence is checked.

\subsection{Voltage Regulators and Shunt Capacitors}

The voltage regulators are widely used in Brazilian networks, which often are long and including urban and rural areas within the same feeder. These regulators usually have variable tap which is defined by Power Flow algorithm through these parameters and characteristics of the regulator control: PT ratio (potential transformer), UR and UX (resistance and reactance to the load center, in the case of regulators with line drop compensation), number of available tap's, connection type and the reference voltage. The voltage regulator output is calculated during the power flow algorithm considering all of these parameters plus the instant values of node voltage and current.

This is an approximate method for predicting the current regulator tap. It is known that the tap switching causes an equipment wear, this effect makes necessary to take other actions to prevent an excessive number of switching as the establishment of insensitivity levels and time delay. The prediction of current tap, because of these features becomes much more difficult, so for real time application of the power flow the better case is to read this parameter remotely via a SCADA system, which needs to be integrated into the Power Flow algorithm.

Shunt capacitors are also used for voltage control through reactive compensation and has important influence on the Power Flow. These are modeled as reactive power injections of fixed value and can be connected or not to the network, depending on the current value in that node or the time of day, in the case of having an automatic control module.

\subsection{Meshed Networks}

The networks that have loops cannot have the Power Flow algorithm based on backward/forward sweep applied directly. Under this condition the algorithm should enter into an infinite recursion, causing a stack overflow.

In these cases the network must go through an initial phase where breakpoints are inserted in order to transform the meshed network into an equivalent radial network. At the breaking point is inserted a bus with a current injection calculated by the method of impedance compensation at the breaking point which is discussed in [4] [8].

\section{Parallel Power Flow}

\subsection{Parallel Computing}

The evolution of microprocessors few years ago, has encountered a limit on clock frequency which keep them from work above a certain frequency range. Therefore the evolution of processing capability is giving by optimizing the circuits and multiprocessing, which is to include more than one processor on a single chip. The multiprocessing capability is spreading among the processors for all types of applications and software developed can use this feature reducing its running time through parallel programming.

The proposed methodology for the parallelization of the power flow algorithm can be applied to any other algorithm of forward/backward sweep into a radial network.

\subsection{Implementation Principles}

An algorithm based on backward/forward sweep can operate in a general way with two main functions:

1) Prefixed Action: is the action that is performed on each node during the step of forward sweeping. Should accept parameters, which are processed and transmitted for processing at the downstream nodes.

2) Postfixed Action: is the action that is performed on each node during the backward sweep. The processing should be performed on the node and the values of interest should be returned to the upstream nodes. As a node can have multiple branches, should also be provided an algorithm to operate over the values of the branches, before returning to the parent node.

The separation of these two elements allows the implementation of the parallelism independently of the algorithm implementation (which is basically composed by prefixed and postfixed actions). The basic principle of the parallel implementation is that from the trunk path of the feeder, all the ramifications can be computed in parallel.

This implies that the feeder can be broke into many feeders and the Power Flow can be solve for that feeders in a parallel way. The Power Flow also must be calculated into the main path and this calculation is responsible by the synchronization between ramifications.

In the first step the algorithm should find the main path of the feeder. This can be determined as the path from the substation to a leaf node (in the end of the network) with the greatest number of hops (Figure 1). Another task of preparation is create slave workers, who will be responsible for processing actions (prefixed and postfixed). The number of slaves created will be equal to the number of the system available processors minus 1 . 
The master worker is the agent responsible for coordinating all the actions of slaves, including the ramifications to be processed. This is made by sending voltage values to the ramifications in the forward sweep and receiving the current amount while is in the backward sweep.

The algorithm of the master worker consists of forward sweeping in the main path of the feeder. When it finds a ramification, that ramification will be transferred to a free slave with the needed parameters (voltage) for power flow calculations. The master worker keeps its forward sweep and when it reaches the last node of the trunk, starts to backward sweep, then the master will operate on the results of branches (sum of the currents).

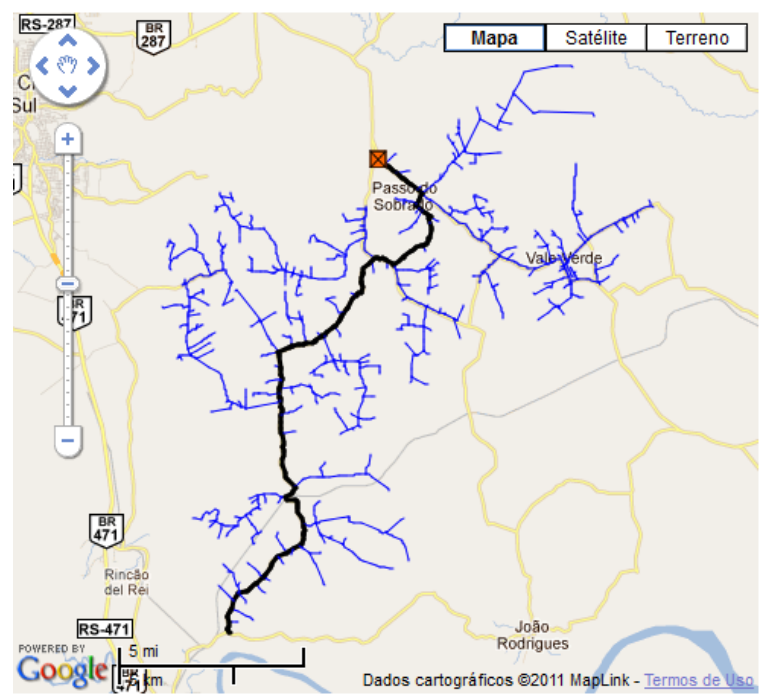

Figure 1: Main path in a typical Brazilian distribution network with 4,132 branches.

\subsection{Load Balancing}

The dispatch of the work from the master worker to the slaves is accomplished through a job queue and not directly to them (Figure 2). Thus the first slave who is free can get a job from the job queue. The use of this approach avoids the use of special load balancing strategies. However, it is necessary to observe the size (in nodes, or hops) of the main path, since a very large path can strain the master worker, which would cause a worse performance of the parallel algorithm.

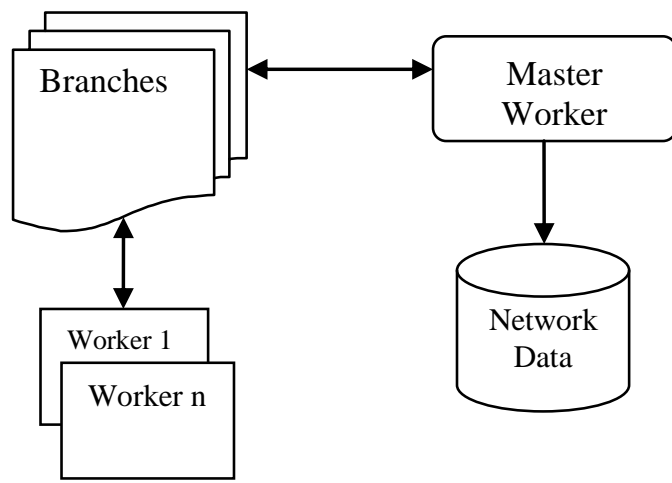

Figure 2: Information flow between the actors of parallel power flow.
Therefore, during the stage of discovering the main path, the algorithm compares the number of nodes of this path relative to the number of nodes in the feeder and the number of workers. With the goal of find a balance between the work of master and slaves, the end of the main path can be transformed into a new branch, which cease to be part of the that path and will be processed by one of the slaves.

This approach allows the algorithm to adapt regardless of the topological characteristics of each network, in order to obtain an adaptive balancing and trying to get the most processing power from the available processors.

\section{Results}

\subsection{Simulations}

The final algorithm was tested using real distribution networks of the CEEE-D, located in southern Brazil. The feeders had 396, 679, and 2517 nodes and another variable in the tests was also the number of available processors that were 2,4 and 6 .

To demonstrate the performance improvement obtained from the parallel algorithm, the power flow was executed on the testing networks in serial and parallel version. The performance values showed in the Figure 3 are the execution time relation between the parallel and the serial implementation. It is possible to realize that the parallelism becomes clearly more efficient as the network nodes were increased. This feature is quite common in Brazilian distribution networks outside of the major urban centers. The algorithm, in the tests performed always was faster than the original, and due to load balancing obtained a performance which could be a linear function of the number of processors.

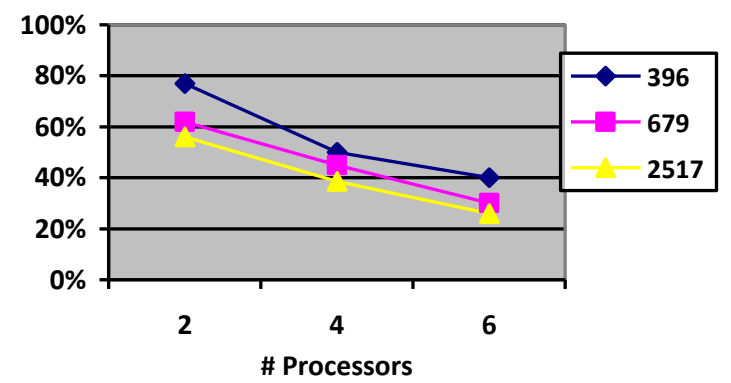

Figure 3: Ratio between the runtime of the serial and parallel algorithm according to the number of nodes and processors.

\subsection{Software Tool}

The algorithms were developed in Java language using a multi-tiered model, where the server tier, which is effectively responsible for processing the Power Flow calculation can be hosted on a powerful multi-processor computer. The client tier technology was developed using Google Web Toolkit ® (Figure 4), which allows visualization of the topology and operation of the 
Software in any Internet browser, or even from smartphones and tablets.

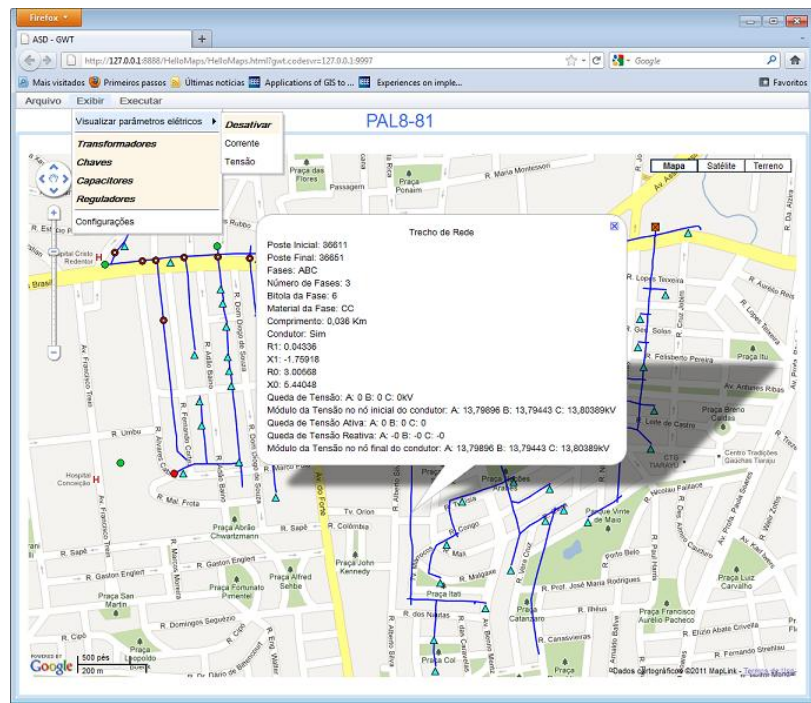

Figure 4: Web based Software Tool

In addition to the real-time characteristic, which could be obtained through the parallel algorithm, ubiquity is also a desirable feature since the popularization of devices such as smartphones and tablets. This multi-tiered model allows that the availability on many devices does not adversely affect the runtime of the application.

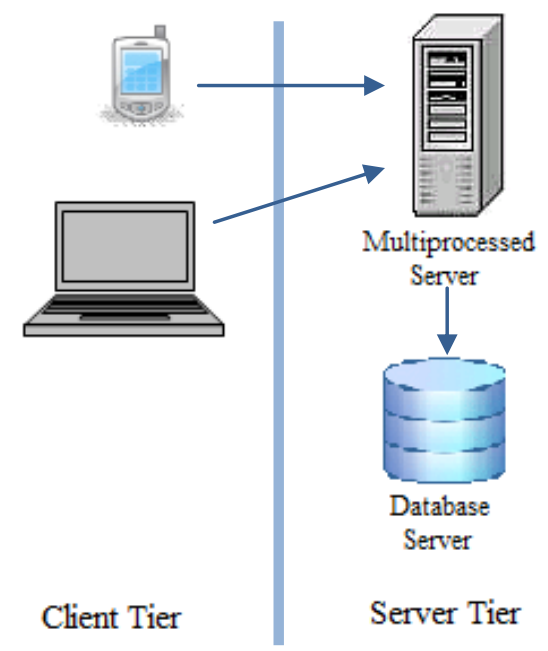

Figure 4: Multi-tier Model

\section{Conclusions}

The presented paper proposes the use of a parallel algorithm in order to implementing the Power Flow in real time even in distribution networks with large numbers of nodes. The reduced time execution will allow the integration of these algorithms to remote measurements along the distribution network as well as measurements of loads on the smart metering devices. The proposed methodology for parallelization was applied to the classic backward/forward sweep power flow algorithm, and also can have execution times even lower if applied to some recent improvements proposed in [8].
Using the methods discussed in [6] [7] was also possible to model the sources of distributed generation in primary distribution networks. Finally, the modeling of multi-tier application brought great advantages as the possibility of real-time execution of complex algorithms even in devices with low processing power.

\section{Acknowledgments}

The authors would like to thank PPGEE, UFSM, CAPES and CEEE-D for the financial support provided to this project and also "ANEEL - Agência Nacional de Energia Elétrica" through the program of R \& D - Research and Development.

\section{References}

[1] W. F. Tinny and C. E. Hart, "Power Flow Solution by Newton's Method", IEEE Trans. on Power Apparatus and Systems, Vol. PAS-86, No. 11, pp. 1449-1460, November 1967. [2] B. Stott and O. Alsac, "Fast Decoupled Load Flow", IEEE Trans. on Power Apparatus and Systems, Vol. PAS-93, No. 3, pp. 859-869, May/June 1974.

[3] M.S. Srinivas, "Distribution Load Flows: A Brief Review", Proceedings of the 2000 IEEE PES Summer Meeting, Singapore, January 2000.

[4] D. Shirmohammadi, H. W. Hong, A. Semlyen and G. X. Luo. A Compensation-based power flow method for weakly meshed distribution and transmission networks. IEEE Transactions on Power Systems, v. 3, n. 2, p. 753-762, May 1988.

[5] R.P. Broadwater, A. Chandrasekaram, C.T. Huddleston, and A.H. Khan, "Power Flow Analysis of Unabalanced Multiphase Radial Distribution Systems", Electric

Power System Research, vol. 14, 1988.

[6] C. Cheng, D. Shirmohammadi. A three-phase power flow method for real-time distribution system analysis. IEEE Transactions on Power Systems, v. 10, n. 2, p. 671-769, May 1995.

[7] S.M. Moghaddas-Tafreshi, E. Mashhour. Distributed generation modeling for power flow studies and a three-phase unbalanced power flow solution for radial distribution systems considering distributed generation. Electric Power Systems Research, Vol. 79, Issue 4, p. 680-686, April 2009.

[8] G. Chang, S. Chu, M. Hsu, C. Chuang, H. Wang. An efficient power flow algorithm for weakly meshed distribution systems. Electric Power Systems Research, Vol. 84, Issue 1, p. 90-99, March 2012. 